\title{
Conocer las rutinas para innovar en la geografía escolar ${ }^{1}$
}

\section{To know the routines to innovate in school geography}

\author{
Xosé M Souto González y Diego García Monteagudo²
}

\begin{abstract}
RESUMEN
Muchas propuestas de innovación fracasan ante la permanencia de las rutinas escolares y las tradiciones educativas. La teoría de las representaciones sociales nos permite conocer dichos obstáculos, que se insertan como hábitos profesionales en la enseñanza de la geografía. En este artículo mostramos la convergencia de las metodologías de las representaciones y la geografía de la percepción y del comportamiento en el análisis de la geografía escolar. Los ejemplos empíricos con diferentes sujetos de los que hemos obtenido información nos muestran la posibilidad de transformar los obstáculos en problemas y diseñar una alternativa educativa que conceda una utilidad social a la geografía escolar.
\end{abstract}

Palabras clave: Representaciones sociales, geografía de la percepción, rutinas escolares, enseñanza de la geografía, innovación

\begin{abstract}
Many proposals for innovation fail due to the permanence of school routines and educational traditions. The theory of social representations allows us to know these obstacles, which are inserted as professional habits in the teaching of geography. In this article we show the convergence of the methodologies of representations and the geography of perception and behavior in the analysis of school geography. The empirical examples with different subjects from which we have obtained information show us the possibility of transforming obstacles into problems and designing an educational alternative that grants a social utility to school geography.
\end{abstract}

Key words: social representations, Behavioural and Perception Geography, school routines, geography teaching, innovation.

Este artículo se inserta en la línea de investigación del proyecto "Competencias sociales para una ciudadanía democrática: análisis, desarrollo y evaluación". Comdemo (Red 14) dentro del marco del Plan Nacional de I+D+i del Gobierno de España y de los Fondos FEDER de la UE (EDU2015-65621-C3-1-R); además del titulado "Las marginaciones personales y la utilidad social del saber escolar" y referencia GVAICO2016-092, en el marco de la convocatoria de la Conselleria d’Educació, Cultura i Esports para Ayudas para Grupos de Investigación Consolidables (Orden 6/2015, de 9 de septiembre) y que continua en el Proyecto de Título: Las representaciones sociales de los contenidos escolares en el desarrollo de las competencias docentes, Proyectos I+D de Generación de Conocimiento y Fortalecimiento Científico y Tecnológico del Sistema I+D+i (Ministerio de Ciencia, Innovación y Universidades), con referencia PGC2018-094491-B-C32.

Universitat de València y proyecto Gea-Clío 
Las rutinas escolares se construyen a lo largo de varias generaciones y resisten con fuerza el impacto de las innovaciones educativas. En España en el último cuarto del siglo XX, las transformaciones políticas acaecidas tras la muerte del dictador Franco, las modificaciones tecnológicas de la comunicación de masas y la irrupción de nuevas concepciones educativas (teorías y evaluaciones internacionales) parecía que podrían acabar con ciertas rutinas existentes en la enseñanza y aprendizaje de las ciencias sociales. Sin embargo, las estadísticas y, sobre todo, el comportamiento del alumnado en las aulas del sistema escolar nos indican que la mejora de la educación es todavía una quimera.

En el caso de España los cambios legislativos y la transición de la dictadura franquista a la democracia constitucional supusieron cambios en la organización territorial, que afectaron a las competencias en la educación (Pérez y Souto, 1989: 36-37). La aparición de las Comunidades Autónomas repercutió de dos maneras diferentes en la enseñanza de la geografía. Por una parte, en la selección de los contenidos educativos (hechos locales, actitudes de identidad); por otra, en la organización del currículo específico y la regulación de las condiciones laborales de los docentes. Un doble condicionante institucional que afecta a la explicación de la geografía escolar. En el caso que nos ocupa vamos a estudiar el caso de la Comunidad Valenciana (Figura No 1), donde hemos realizado los estudios empíricos con centros escolares de enseñanza secundaria y con la formación inicial en la Universitat de Valencia ${ }^{3}$.

Figura No1.

España y sus comunidades autónomas: la comunidad valenciana

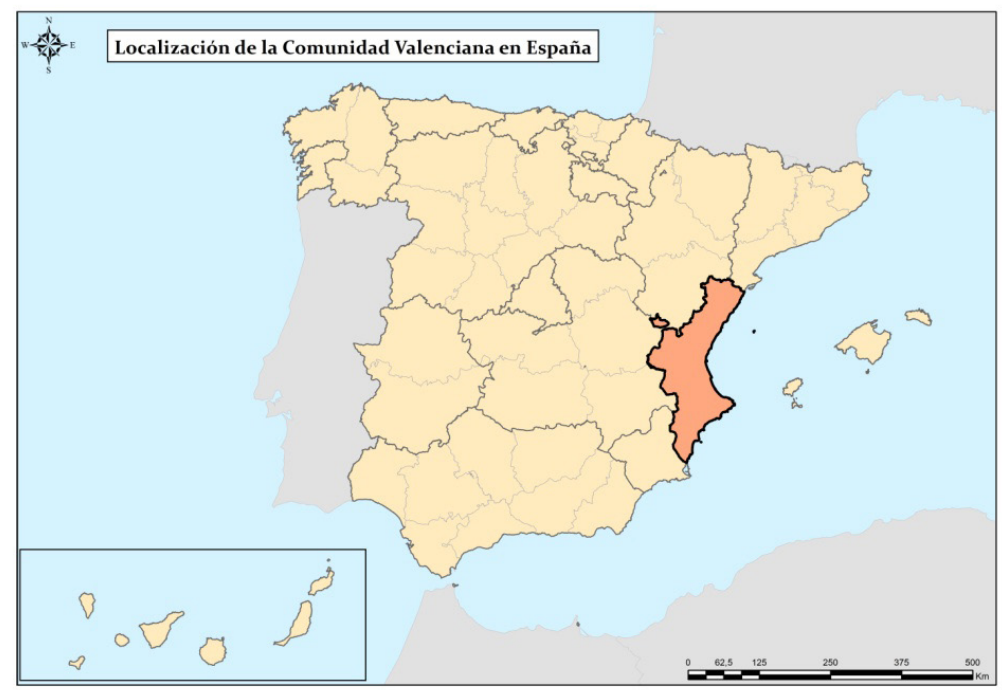

Fuente: Elaboración propia.

Hemos analizado las opiniones del alumnado que se formaba para ser profesorado de Educación Primaria (Grado de Maestro/a en Educación Primaria) como para ser docente en Educación Secundaria (Máster de Formación del Profesorado en Educación Secundaria). 
Los cambios acaecidos en los medios y técnicas de la información de masas, las interferencias culturales de lugares lejanos, así como las nuevas maneras de relaciones sociales inciden en las nuevas explicaciones que realizan las personas sobre la geografía del planeta en sus diferentes escalas. Sin embargo, la metodología y los contenidos escolares no siempre acompañaron a estas transformaciones. Sabemos que las estrategias de enseñanza tienen una finalidad básica: facilitar el aprendizaje de los hechos sociales en unas personas que tienen unas emociones, actitudes ante el conocimiento escolar y capacidades cognoscitivas determinadas. $Y$ por eso la investigación educativa de las didácticas específicas se ha esforzado en presentar nuevas maneras de enseñar para favorecer un aprendizaje significativo (Carretero et al., 1995; Coll et al., 1993; Driver, 1988; Gil-Pérez, 1994). Unas propuestas que buscaban un modelo de enseñanza para orientar la praxis, que, sin embargo, se diluyó en multitud de propuestas prácticas en las primeras décadas del siglo XXI como consecuencia, en gran medida, de la irrupción de las $\mathrm{TIC}^{4}$, que incluso generó nuevos mitos de información y una apariencia de dominar la información.

Las intuiciones que tienen los docentes de este nuevo milenio sobre las modificaciones indican que existen cambios formales en el uso de las tecnologías informáticas y en los manuales escolares, pero la comunicación escolar sigue siendo semejante a la de los años cincuenta y sesenta del pasado siglo. Los debates mantenidos acerca de la incidencia de las nuevas leyes en el fracaso escolar adolecían del rigor necesario y era más una manifestación social del sentido que se le concedía a la educación escolar que un análisis del aprendizaje realizado para poder ejercer la ciudadanía en la sociedad5. En el caso de la geografía escolar, la irrupción de técnicas digitales de localización y los sistemas de información geográfica (SIG) creó la ilusión de un cambio en la manera de enseñar y aprender los problemas geográficos en sus diferentes escalas planetarias.

Transformar dichas intuiciones, a veces erróneas, en hechos argumentativos implica recurrir a la teoría de las representaciones sociales, pues nos permiten comprender no sólo qué idea existe sobre la cultura escolar, sino más propiamente sobre las relaciones entre ésta y la educación geográfica. Entendemos que esta teoría nos permite explicar las resistencias u obstáculos epistemológicos que aparecen en el momento de programar una innovación escolar. Precisamente parafraseando a Gastón Bachelard (1987) podemos concluir que en el estudio de la realidad, "lo que cree saberse con claridad, ofusca lo que debería saberse"; es decir, los prejuicios sobre una situación impiden su planteamiento como problema científico. Y en el caso de la enseñanza de la geografía y de otras ciencias sociales, dichos estereotipos se han naturalizado de tal manera que hace muy difícil la innovación. Además en el caso de las representaciones sociales, como se dirá, existe una convergencia teórica con otros modelos explicativos que aparecen en las ciencias de la educación y en la propia geografía.

La estructura de este artículo parte de la transformación del obstáculo cotidiano (el comportamiento escolar) en un problema de investigación. Como tal existirá una metodología con sus hipótesis de resolución. Con los datos extraídos de encuestas y grupos de discusión pretendemos

La revista Ara@ne, dentro de la plataforma Geocrítica de la Universidad de Barcelona, publica mensualmente novedades sobre las TIC y el conocimiento geográfico, analizando críticamente su irrupción en la opinión pública.

A este respecto se pueden consultar diferentes artículos y debates periodísticos sobre los niveles educativos en España. Por ejemplo el artículo de José Saturnino Martínez, profesor del departamento de Sociología de la Universidad de La Laguna en el diario El País el 21 de noviembre de 2005: ¿Baja el nivel educativo? También el blog iberoamericano Geocritiq, dentro de la plataforma on-line de Geocrítica (Universidad de Barcelona) ha publicado otros artículos sobre el mismo contenido. 
ofrecer una justificación empírica de las características que determinan la geografía escolar en este siglo XXI y las posibilidades de su innovación en la praxis educativa.

\section{El problema del aprendizaje social y las teorías explicativas}

Las representaciones nos permiten conectar las realidades vividas por el sujeto con la socialización que se produce en un centro escolar, como también han validado en su estudio empírico dos profesoras colombianas. "En el caso específico del ámbito escolar, la socialización está vinculada a la subjetividad porque en ella se produce una puesta en cuestión a la naturalización del orden mediante la reivindicación de derechos, que posibilitan nuevos sentidos para significar las relaciones sociales" (Palacios y Herrera, 2013: 432). Este estudio y otros realizados en España ${ }^{6}$ nos señalan que la dicotomía entre sujeto/objeto no era la más adecuada para diagnosticar los aprendizajes que se utilizaban para decidir con autonomía en el medio social. Los contextos institucionales y las ideas hegemónicas en la opinión pública influyen en el momento de programar las actividades escolares.

Los procesos de naturalización de ciertos procesos históricos, así como la referida a las capacidades que se consideran innatas del individuo para aprender la cultura; o bien la concepción de la geografía escolar como un conjunto de contenidos canónicos que nos revelan la armonía del paisaje de acuerdo con las expectativas humanas, son consecuencia de productos creados por los seres humanos. Por eso queremos mostrar un conjunto de evidencias de la praxis que nos permitirán plantear la posibilidad de transformar los obstáculos en problemas de investigación y de este modo buscar una hipótesis de resolución que nos permita innovar en la educación geográfica.

Para desarrollar este proceso argumentativo hemos procedido a focalizar el problema en el siguiente enunciado: los recuerdos de las clases recibidas actúan como guión de vida que organizan las pautas de comportamiento docente. Ello supone preguntarse al menos las siguientes cuestiones: ¿Qué nos dicen los docentes y futuros profesores acerca del contenido escolar de la Geografía? ¿Por qué se repiten ciertos estereotipos a lo largo de diversas generaciones? ¿Por qué perdura una cierta representación social del saber geográfico escolar? Unas preguntas que nos remiten a respuestas verbales que están teñidas de emociones y sentimientos, que sin duda organizan un contexto moral que, como veremos, se manifiesta en el momento de pensar en la enseñanza de la geografía.

Desde la perspectiva del análisis de la docencia en la Geografía escolar nos interesa la aportación de las representaciones sociales como síntesis de las influencias sociales e individuales en la forma de trabajar del profesorado, que, como sabemos por otros estudios desde la enseñanza de la Historia, es un elemento esencial para el agrado o desinterés del alumnado por la materia?.

Un caso significativo es el grupo IRES de Andalucía, que viene desarrollando una amplia investigación en España desde hace treinta años sobre el papel de los profesores en el conocimiento escolar.

Sobre estas cuestiones hemos desarrollado una larga investigación en relación con los recuerdos escolares de la Historia de España (Souto, Martínez y Parra, 2014) 
La idea que tiene de la enseñanza escolar es un factor decisivo para una actitud de conocimiento, pues sin ese afán de querer conocer no es posible que se produzca un aprendizaje significativo.

La Geografía escolar se elabora en un marco institucional y en unos espacios regulados por leyes, que interfieren las relaciones entre sujetos. La Tesis doctoral de la profesora colombiana Nancy Palacios (2018) nos advertía de la interpretación de derechos y deberes por docentes y alumnos en los centros escolares seleccionados. Y nos explicaba con claridad cómo su regulación afectaba a la construcción del conocimiento. En esta misma línea se manifestaba J. Saraiva (2007) para explicar, también en una Tesis doctoral, los motivos de la elección de la profesión de docente de Geografía en la enseñanza fundamental en el Norte de Brasil; un estudio que se encuadraba en las líneas de investigación de M. Domingos (2000, 2010).

Nuestro interés por este programa de investigación reside en que nos relacionan el análisis psicológico de S. Moscovici con el sociológico de P. Bourdieu, en la búsqueda de la explicación del habitus docente en la forma de enseñar la Geografía escolar. En este sentido, influyen las características de origen social y familiar del propio docente, lo que determina su concepción del sistema escolar; que influye decisivamente en su formación inicial y continua, con las lecturas que pueda realizar, los seminarios de trabajo que pueda fomentar y los debates con otros colegas. Un contexto social donde se forma la idea de la Geografía escolar, donde los hechos conceptuales se proyectan a los espacios y momentos de la comunicación entre alumnos y docentes. Las opiniones individuales se construyen, de este modo, condicionadas por el contexto social.

Entendemos que el marco teórico de las representaciones sociales es el más apropiado por dos motivos fundamentales. En primer lugar, porque nos explica las razones de los sujetos para actuar de una manera determinada, en este caso cuando enseñan geografía, respecto a unas ideas que han construido individualmente, pero en un proceso histórico concreto. En segundo lugar, porque los planteamientos de las representaciones sociales nos permiten averiguar las concepciones espontáneas de los alumnos y alumnas, lo que nos permite impugnar las ideas del sentido común en las aulas de la enseñanza básica.

Además influyen los cambios teóricos de las ciencias de referencia (en este caso la geografía con sus tendencias o escuelas), de las ciencias del aprendizaje (el constructivismo y las teorías neurocientíficas) y los estudios que analizan las relaciones entre la objetividad de los hechos sociales y la actuación del sujeto (como la sociogénesis o las representaciones sociales). Es una teoría que nos permite explicar con más rigor que otras, por ejemplo la trasposición didáctica, las diferencias entre los conocimientos académicos y la construcción del saber escolar.

Finalmente está el comportamiento discente. Existe en las personas, que conocemos como alumnos, un hábito de comprensión vulgar del mundo que genera explicaciones "ad hoc". Y en esta manera de comprender y explicar el mundo se impone la naturalización de ciertos hechos sociales. Una manera de reflexionar que genera un comportamiento ciudadano, con sus justificaciones morales, que más de una vez se transforma en una explicación exculpatoria.

Este panorama se puede analizar con la ayuda de los modelos que se han establecido desde el último cuarto del siglo XX en la esfera de las ciencias sociales, como es la Geografía y la Educación. En el primer caso el denominado "giro del comportamiento" (Capel, 1973) surge como 
respuesta al fracaso del neopositivismo de la geografía cuantitativa en su esfuerzo por explicar las desigualdades en las identidades espaciales y en la ordenación del territorio. La interpretación fenomenológica de los hechos humanos abrió una línea explicativa que se acotaría en los métodos de la Geografía Humanística y en los de la Geografía de la Percepción y del Comportamiento (Boira y Reques, 1991). Las emociones, las intenciones y los prejuicios aparecen como factores explicativos de las decisiones sociales en la apropiación del espacio.

En el caso de las ciencias de la educación emana de la convergencia de los planteamientos de la sociología, como son los estudios de Bourdieu y Passeron (1979) con los que surgen en el seno de la psicología y, con posterioridad de la neurociencia (Díaz, 2007; Damasio, 2010). En esta convergencia se han basado las investigaciones de la didáctica específica (Driver, 1988; Gil-Pérez, 1994, Souto, 2013) y en el caso específico de las disciplinas escolares ha preocupado su desarroIlo histórico (Goodson, 1995; Cuesta, 1997). En su conjunto dichas pesquisas ponen de relieve que las materias escolares se corresponden con decisiones sociales que se imponen como naturales y que son asumidas como tales por los docentes; ello conduce a considerar cualquier materia escolar como un singular único, sin entender la diversidad de opciones que existen en el campo académico para organizar las actividades curriculares. Frente a ello solo cabe estudiar los fundamentos epistemológicos de la materia de referencia, así como las ciencias de la educación, que la sistematizan como disciplina escolar, y las representaciones sociales que la sustentan.

En resumen, este modelo surge de la convergencia de los planteamientos sociológicos con el constructivismo en el aprendizaje humano y las experiencias de la investigación académica de la geografía de la percepción y del comportamiento. Es decir, desarrolla el habitus de Bourdieu en las relaciones sujeto y contexto social, las propuestas de Vygotsky sobre el aprendizaje cultural, y la impugnación de los estereotipos y prejuicios, que nos pone de relieve la investigación geográfica sobre los territorios.

Así pues, este cambio en las concepciones de los principios que articulaban la explicación de un territorio desde la geografía coincidía con las transformaciones existentes en los principios de aprendizaje. Son especialmente relevantes dos procesos que inciden en las didácticas de las ciencias sociales; por una parte, la modificación de las teorías existentes sobre los test de inteligencia (de Binet) que se ven impugnados desde la teoría de las inteligencias múltiples (de Gardner), aunque tampoco podemos caer en la seducción de esta teoría como panacea (Rangel, 2010). Por otra parte, la irrupción de las teorías de Piaget y Vygotsky en la interpretación de los aprendizajes sociales, donde no sólo influía la capacidad de adaptación cognoscitiva, sino que los factores culturales y la tensión emocional de la zona de aprendizaje próxima estimulaba a la persona para aprender sobre nuevas situaciones. Las consideraciones anteriores nos indican que el proceso de construcción de explicaciones sociales es una tarea compleja y evolutiva y que debemos cuestionarnos las justificaciones que realizamos en las argumentaciones, donde el papel del sujeto es determinante.

En los primeros decenios del siglo XXI los avances en las investigaciones de la neurociencia (Díaz, 2007; Damasio, 2010) avalaron desde evidencias empíricas la influencia de las emociones y sentimientos en los razonamientos y decisiones individuales y colectivas. Igualmente el estudio de las representaciones sociales (Moscovici, 2011; Jodelet, 2001; Gustavson y Selander, 2011) nos pone de manifiesto que la interpretación que se realiza del sistema escolar está muy mediatizada 
por las expectativas personales de ascenso social o por las creencias de la inutilidad del mismo para un futuro laboral y personal.

También en el siglo XXI inciden con fuerza las nuevas teorías escolares burocráticas, como son las competencias educativas $y$, sobre todo, el intento de medir los aprendizajes realizados en la escuela. Sin embargo, coincidimos con autores como Julio Carabaña, que muestra con datos empíricos como muchas de las actividades realizadas en las pruebas externas miden realmente lo que pueden medir y no tanto lo que se desea. Así, respecto al Informe Coleman señala que "eligió dejar fuera los conocimientos, las destrezas y la capacidad de resolver problemas particulares que son el objeto específico de las escuelas y que son cada vez más importantes en el trabajo y en las relaciones sociales" (Carabaña, 2015: 27).

\section{El estudio metodológico de las rutinas escolares}

Con todos estos precedentes hemos querido incidir en el mundo de las representaciones sociales de la geografía escolar a través de los datos empíricos obtenidos en las prácticas escolares. Para ello hemos efectuado diferentes estudios entre alumnos de formación para ser profesores de Educación Primaria o Secundaria en el área de ciencias sociales, geografía e historia. Por una parte, las encuestas de preguntas abiertas buscando la asociación libre de ideas con las palabras geografía y profesor de geografía. En un primer momento trabajamos con la cuantificación manual de las respuestas, mientras que más tarde utilizamos el programa EVOCATION 2005 para determinar las ideas que forman parte del núcleo central de las representaciones sociales, según el planteamiento de Abric (1994). Por otra parte, el debate en clase a modo de grupo de discusión. El trabajo realizado con estas muestras aleatorias nos ha permitido analizar críticamente las técnicas y métodos utilizados en otras investigaciones semejantes. Así hemos sido conscientes de la necesidad de delimitar muy bien los sujetos en sus colectivos: por una parte eran profesores en formación continua (grupo de Cali, docentes de Valencia y Porto Alegre) que deseaban participar en esta actividad, que les resultaba gratificante y, por otra, futuros docentes que veían la tarea como una más de su formación ${ }^{8}$. Por eso el nivel de compromiso y debate no es semejante en los colectivos analizados.

En consecuencia, hemos clasificado las respuestas en tres grupos: docentes en activo, futuros docentes de Educación Primaria (Grado) y Educación Secundaria (Máster) (Cuadro No 1). Con ello queremos mostrar la incidencia del contexto de actuación en la formulación de las ideas, lo que también pone de relieve las semejanzas y permanencia de las rutinas. Igualmente los debates posteriores nos han servido para profundizar en las palabras seleccionadas.

Las fechas de los cuestionarios y posterior debate en clase han sido los siguientes. En la formación continua: Porto Alegre, Brasil (31/8/2015); Cali, Colombia (08/10/2015); Valencia, España (04/03/2016). En formación inicial se corresponden con cursos del Máster de Formación de Profesorado en Educación Secundaria de la Universidad de Valencia (22/10/2015; 25/10/2016 y 17/10/2017); así como el Grado de Educación Primaria de la misma Universidad: 14/09/2015 y 29/10/2015. Las sesiones de debate tenían una duración de dos horas. 


\section{Cuadro No 1.}

Palabras asociadas a profesor/a de geografía

\begin{tabular}{|c|c|c|}
\hline \multicolumn{2}{|c|}{ Grupo de personas } & Palabras o frases asociadas \\
\hline Perfil de la muestra & Contexto y aclaraciones & Ejemplos y frecuencia $(n)$ \\
\hline $\begin{array}{l}\text { Docentes en activo } \\
(\mathrm{N}=68) \text { de Porto Ale- } \\
\text { gre, Cali y Valencia. } \\
2015 / 16\end{array}$ & $\begin{array}{l}\text { Docentes en activo y con deseo } \\
\text { de participar. Existe un compro- } \\
\text { miso social de los sujetos. }\end{array}$ & $\begin{array}{l}\text { Interdisciplinares (8), Dinamismo (7), } \\
\text { Reflexivo (7), Creativo (6), Didáctica } \\
\text { (5), Paciencia (2), Saber enciclopédi- } \\
\text { co (2), Motivación (3), Actualidad (3). }\end{array}$ \\
\hline $\begin{array}{l}\text { Máster de Formación } \\
\text { de Secundaria (N= } \\
\text { 107). } 2015 \text { a } 2017\end{array}$ & $\begin{array}{l}\text { Comparación de resultados in- } \\
\text { dividuales y agrupamiento en } \\
\text { cinco grupos de cuatro perso- } \\
\text { nas. Las palabras se clasifican } \\
\text { en positivas y negativas para su } \\
\text { debate en grupos de discusión. }\end{array}$ & $\begin{array}{l}\text { Práctico-metódico-analítico (20), Di- } \\
\text { námico (12), Carismático (7), Actuali- } \\
\text { zado e informado (4), Crítico, respe- } \\
\text { tuoso, empático (4); Pasmo-aburrido } \\
\text { (10), Teoría/espeso (6), Tradicional/ } \\
\text { monótono (4); Profesionalidad do- } \\
\text { cente (6), Pasión (3), Motivación (2), } \\
\text { Educador (2), Interés (3), Investiga- } \\
\text { dor (2), Transmisión (2), Interdiscipli- } \\
\text { nar (3). }\end{array}$ \\
\hline $\begin{array}{l}\text { Grado de Magisterio } \\
(\mathrm{N}=65) .2015\end{array}$ & $\begin{array}{l}\text { Debate posterior para compren- } \\
\text { der el significado de cada pala- } \\
\text { bra. Dinamismo se asociaba a } \\
\text { actividad práctica (tareas ma- } \\
\text { nuales). }\end{array}$ & $\begin{array}{l}\text { Dinamismo (18); Motivación (11); His- } \\
\text { toriador (5); Conocimiento (4); Abu- } \\
\text { rrido (4); Comunicador (4); Leer libro } \\
\text { (3); Memorizar (3), Adoctrinador(2); } \\
\text { exámenes (2) }\end{array}$ \\
\hline
\end{tabular}

Fuente: Encuestas, cuestionarios y grupos de debate. Elaboración propia

Tal como podemos comprobar existe una gran dispersión de conceptos en la selección de palabras asociadas al concepto de profesor de Geografía. Se alude de forma mayoritaria a una serie de términos que podemos interpretar como genéricos: dinámico, profesional, metódico, motivador... Es decir rasgos generales del comportamiento, por lo que podemos apreciar que el alumnado en formación, así como algunos docentes que ejercen la profesión, destacan sobre todo la relación comunicativa que se establece con el alumnado y, en menor medida, el contenido que imparte, que se ve desde una posición de conocimiento globalizador. Resulta evidente, sobre todo después de los debates en los grupos de discusión, que los encuestados no diferencian la práctica escolar de la praxis; tal parece como si la labor del profesor fuera la de un prestidigitador que sorprende a sus alumnos. Por el contrario, destaca el vocablo "reflexivo", que está más presente en el caso de los docentes en ejercicio que en los que están formándose; igualmente el concepto interdisciplinario aparece con más frecuencia en el profesorado en ejercicio, pues según han indicado la explicación geográfica implicaba un diálogo con otras materias (Historia, Ciencias Naturales, Lengua).

Parece que podemos avanzar la hipótesis que indica que la representación social del docente innovador de Geografía está relacionada con una visión desde el sentido común y que es aceptada 
como tal por el profesorado. Es decir, existe una cesura entre lo que se expone en el conocimiento académico y lo que se transmite como profesión o difusión de este conocimiento específico, que se entiende como la aplicación o instrucción de dicho saber. Incluso cuando se analizan moralmente los conceptos (palabras positivas/negativas) hemos comprobado cómo el comportamiento comunicativo es la clave de dicha clasificación, pues se revela un comportamiento moral y no ético. Así los ejemplos de palabras como monótono, espeso, pasmo, aburrido manifiestan el agrado/desagrado en el recuerdo de las clases que tenía el alumnado con sus profesores, pero no su razonamiento respecto a la forma de explicar el docente. Menos común era la imagen del docente de la enseñanza básica en contacto con los centros universitarios para poder estudiar o reflexionar sobre cómo se produce el aprendizaje humano sobre los hechos sociales en el espacio geográfico (como podemos apreciar el concepto "investigador" aparece pocas veces); una afirmación que ya había sido demostrada en el curso de investigaciones anteriores sobre la enseñanza de la geografía (Ramiro, 1998).

Ello se ve reforzado cuando se analiza la concepción espontánea de la geografía escolar. Mapas, territorio, medio ambiente e interdisciplinariedad aparecen en las menciones de las personas que hemos encuestado. Sin embargo en los debates consecutivos y en los análisis de los conceptos utilizados hemos observado una diversidad de apreciaciones y una repetición de ideas genéricas, como era la de considerar que los mapas se corresponden con la realidad geográfica y que ésta está relacionada con el medio ambiente. Preocupan, sin duda, problemas actuales y la habilidad por conocer los hechos en su representación cartográfica, pero cuando profundizamos en su conocimiento, se observan referencias a una cultura superficial que puede ser un doble obstáculo a la innovación. Por una parte, porque no es un contenido motivador para las expectativas del alumnado y, por otra, porque puede suponer una situación de incertidumbre por parte del profesorado, que puede pensar que no domina los elementos teóricos y las habilidades precisas para explicar adecuadamente las situaciones ambientales.

Para poder establecer un análisis más profundo hemos seleccionado las opiniones del alumnado del Máster de Profesor de Educación Secundaria ( $N=107)$ y las hemos comparado con las muestras del profesorado en activo de Perú9 $(\mathrm{N}=88)$ y con los de Grado de Magisterio en Educación Primaria ( $\mathrm{N}=35)$ (Cuadro $\mathrm{N}^{\circ} 2$ 2). En el caso de los docentes de Perú contamos con el análisis complementario realizado por otros compañeros del área de didáctica de las ciencias sociales (Bel Martínez et al., 2017), donde muestran que las imágenes que se transmiten por los medios de comunicación influyen en sus decisiones de visitar o vivir en un lugar y que influye en los equívocos de escala del lugar, como es la confusión entre país y ciudad. Esta influencia del pensamiento dominante interviene en la construcción de las ideas espontáneas por parte del alumnado del Máster de Profesor de Educación Secundaria, pues cuando se analiza el cambio climático no se sabe valorar la influencia del efecto invernadero en la vida de los seres humanos. Ambas conclusiones que parecen obvias no lo son tales en el momento de programar los contenidos escolares y explicar una situación problemática en las aulas. Por eso entendemos que la confluencia de las representaciones sociales y la geografía de la percepción es muy útil para poder llevar adelante una innovación didáctica en el área de la geografía escolar. Sin una reflexión consciente no es

Los docentes de Perú era un conjunto de 88 personas que asistían a un curso de formación en Valencia, con financiación del gobierno de dicho país. Nos ha servido para poder establecer las diferencias y generalizaciones. En el caso de los alumnos de Grado de Maestro/a en Educación Primaria usamos su muestra para contrastar la formación inicial genérica y específica. 
posible impugnar las rutinas que nos impiden avanzar en el aprendizaje de los problemas sociales en el territorio.

\section{Cuadro № 2 .}

Ideas o palabras asociadas a geografía

\begin{tabular}{|l|l|l|}
\hline \multicolumn{2}{|c|}{ Grupo de personas } & \multicolumn{1}{|c|}{ Palabras o frases asociadas } \\
\hline \multicolumn{1}{|c|}{ Perfil de la muestra } & \multicolumn{1}{|c|}{ Contexto y aclaraciones } & \multicolumn{1}{c|}{ Ejemplos y frecuencia (n) } \\
\hline $\begin{array}{l}\text { Docentes en acti- } \\
\text { vo (N=88) de Perú } \\
(08 / 03 / 2016)\end{array}$ & $\begin{array}{l}\text { Formación de docentes de perso- } \\
\text { nas adultas. Temas genéricos de } \\
\text { ciencias sociales }\end{array}$ & $\begin{array}{l}\text { Espacio (56), Mapa (29), Territorio } \\
\text { (20), Localización (19). }\end{array}$ \\
\hline $\begin{array}{l}\text { Máster de Profeso- } \\
\text { rado de Educación } \\
\text { Secundaria (N=107). } \\
2015 \text { a 2017, Tres gru- } \\
\text { pos. }\end{array}$ & $\begin{array}{l}\text { a la geogninio de palabras asociadas física, en especial } \\
\text { con relieve y clima, donde se aña- } \\
\text { co", bajo la influencia de los me- } \\
\text { dios de comunicación }\end{array}$ & $\begin{array}{l}\text { Mapas-cartografía (24) Clima-me- } \\
\text { teorología (11), Relieve (10), Gráfi- } \\
\text { cas (10), Población y Sociedad (21), } \\
\text { Espacio (6), Práctica (6), Entorno } \\
\text { (5) Medio ambiente (17), Territo- } \\
\text { ro (5), Humana (4), Espacio (2), } \\
\text { Geopolítica (2), Tierra (4), Física } \\
\text { (2), Paisaje (3). }\end{array}$ \\
\hline $\begin{array}{l}\text { Grado de Magisterio } \\
\text { (N=35) (29/10/2015) }\end{array}$ & $\begin{array}{l}\text { Las palabras corresponden al re- } \\
\text { cuerdo de sus clases de ciencias } \\
\text { sociales durante Educación Se- } \\
\text { cundaria }\end{array}$ & $\begin{array}{l}\text { Mapas (24), Países (20), Montañas } \\
\text { (13), Ríos (11), Población (9), Clima } \\
\text { (6) }\end{array}$ \\
\hline
\end{tabular}

Fuente: Elaboración propia sobre datos aportados en seminarios de formación docente

Como se dijo, al analizar sus respuestas hemos visto que hay algunas ideas que se repetían, en especial "mapas" o "cartografía". Ello nos condujo a un nuevo método de trabajo: plantear qué entendían por mapa y cómo representaban el mapa del mundo (proyección Mercator, Peters u otra), para mostrar que el mapa era una representación política, no neutra, de la realidad, lo que desconocían en casi la totalidad de los sujetos, pues no se habían planteado el problema de la fidelidad de la representación cartográfica con las superficies del territorio. Igual sucedía cuando se aludía al concepto de migraciones o densidades, donde no se sabían explicar los factores ambientales, históricos y culturales que determinan la distribución de las personas en las diferentes escalas geográficas del planeta. Todo ello confirma los resultados de algunas investigaciones (Fraile-Jurado y López-Martín, 2017) que nos muestran la creación de mitos geográficos en el presente como consecuencia de la rapidez y falta de rigor en la transmisión de informaciones.

La representación de la Geografía como mapas, territorio y espacio, así como la del profesor, asociado a las palabras de dinamismo y motivación, nos ha llevado a buscar una lógica teórica a la interpretación de estas palabras y por eso hemos acudido a las representaciones sociales como teoría que relaciona el contexto social con los argumentos personales. Es decir, buscamos entender los motivos por los cuales un docente de Geografía entiende que su objetivo es guiar el aprendizaje de los problemas sociales y espaciales y no considera necesario conocer las escuelas de 
Geografía, que presentan diferentes maneras de concebir estas situaciones, en especial buscando una complementariedad entre el contexto social y las percepciones individuales. Igualmente deseamos explicar los motivos por los cuales la Geografía se asocia a espacio, territorio y mapas, pero no se utilizan estos conceptos y técnicas para definir los problemas de los lugares donde viven los alumnos o que se perciben a través de los medios de comunicación. Las frases de los alumnos de postgrado ${ }^{10}$ en la Universidad de Valencia resumen bien las ideas que estamos expresando, pues manifiestan los prejuicios existentes respecto a la enseñanza de la geografía escolar:

Quan em pare a pensar en la meua primera idea sobre geografia escolar, em venen a la ment quasi sense adonar-me'n els montons de mapes, tant físics com polítics, els països, les capitals, les muntanyes, els rius, els mars i oceans..., estudiats al llarg de tota la meua escolaritat. (M1, MIDE)

La visión que tenía de la geografía antes de empezar este módulo era muy simple, superficial y conceptual, puesto que cuando pensaba en esta asignatura solo me venían a la mente palabras como: mapas, pirámides de población, más mapas, ríos, montañas, un poquito de sociedad, política y más mapas, eso sí estos últimos que no faltasen (V1 MIDE) ${ }^{11}$.

\section{El aval empírico de la persistencia de las representaciones sociales de la geografía escolar}

En la búsqueda de preguntas para seguir cuestionando la realidad percibida por el alumnado nos encontramos en la siguiente situación. Disponemos de un conjunto de datos (resultados de encuestas, de grupos de debate) que nos revelan una manifestación de la geografía escolar asociada a las rutinas académicas, pero también a la influencia del contexto social. Las resistencias al cambio de los sujetos implicados, como son las familias de los alumnos (Fernández et al., 2010), de los propios docentes (Müller, 1993) o de la institución universitaria (Ramiro, 1998) nos pone de relieve que las ideas se construyen en un determinado contexto social. En el análisis de estas ideas precisamos conocer la opinión de la ciudadanía, para lo cual hemos recurrido a las entrevistas, pues nos permitían profundizar más que las encuestas en el conocimiento de los elementos y factores que determinan la visión hegemónica "natural" de la geografía escolar.

Para conocer cómo se construyen las rutinas escolares que determinan nuestro comportamiento particular disponemos de dos maneras de acercarnos. Por una parte, la historia escolar de la disciplina escolar, siguiendo la estela del proceso metodológico que se ha llevado a cabo en la enseñanza de la historia (Cuesta, 1997; Goodson, 1995) y que cuenta con aportaciones valiosas en el caso español (Capel et al., 1983; Capel et al., 1985; Luis-Gómez, 1985; Melcón-Beltrán, 1995). Por otro lado, el campo de las representaciones sociales, para acercarnos a los factores sociales que conectan el contexto social con la posición del sujeto que enseña la geografía.

10 Nos referimos al Máster de Investigación en Didácticas Específicas (MIDE) de la Universidad de Valencia. Las valoraciones que aparecen corresponden a alumnos/as del curso 2016/17 (redacciones realizadas en enero de 2017)

Para mantener el respeto a la privacidad de las personas entrevistadas hemos colocado V (varón) y M (mujer) para identificar a las personas que formaban parte del curso del MIDE del año escolar 2016/17. 
El estudio de la sociogénesis escolar de la Geografía está determinado, como en el caso de la Historia, por la necesidad de legitimar la configuración de los Estados-nación en el momento de la institucionalización del aparato administrativo. Para ello la Geografía escolar organiza un discurso desde el modelo regional que supone legitimar la organización del Estado con sus diferentes partes (regiones, provincias) en las cuales se define una homogeneidad paisajística, que con el tiempo ha perdurado como elemento estereotipado de una parte del territorio ${ }^{12}$. Este aparece como un escenario estático donde tienen lugar los acontecimientos históricos; por eso en algunos casos la simbiosis de la geografía e historia escolar se resolvía presentando el marco físico del territorio con sus mapas, después transcurría la sucesión de hechos ordenados cronológicamente y finalizaba con un panorama de los hechos económicos más recientes. Una manera de exponer las características del territorio que no implicaba ningún análisis de problemas sociales o ambientales ${ }^{13}$.

Por su parte el análisis de las representaciones sociales nos acerca a las características que podemos conocer cómo la psicología de masas en relación con la posición del individuo (Moscovici, 2011: 83-84). Nos interesaron las afirmaciones de Jodelet (2001) asociando la construcción social del conocimiento con la presentación de una información cultural "distinguida", que se presenta como "natural" y estática, que para los docentes son "los contenidos que deben impartir en las aulas". Son realidades asumidas desde una condición pre-reflexiva y, por tanto, se derivan en comportamientos que no se razonan, se asumen, lo que refuerza la idea de una geografía escolar tradicional. Son emociones y razonamientos que se mantienen estables más allá de las coyunturas históricas, que se corrobora en frases como las siguientes:

La Geografía era únicamente un ejercicio de memoria sin interés: aprender nombres y saber dónde colocarlos, como un puzle (V2 MIDE).

Hablando con sinceridad, la Geografía siempre ha sido una de las pocas asignaturas que nunca me llegó a gustar. Para mí, durante la etapa escolar la Geografía era esa parte aburrida de la que inexplicablemente me hacían examinarme junto con Historia (V3 MIDE).

La representación es "un corpus organizado de conocimientos y una de las actividades psíquicas gracias a las cuales las personas hacen inteligible la realidad física y social, se integran en un grupo o en una relación cotidiana de intercambios, liberan los poderes de su imaginación y castran nuestra creatividad" (Moscovici, 1979:17-18). Esta teoría nos permitía explicar las concepciones espontáneas que veníamos trabajando desde los años noventa del siglo pasado y que hemos sintetizado en la figura $n^{\circ} 2$. La aportación que ahora conocemos es que las representaciones son algo más, un conjunto de ideas que se elaboran en un determinado contexto y por eso es preciso definir muy bien dicho ámbito de elaboración. En el caso de la geografía se elaboran en el medio escolar, bajo la influencia de la idea del sentido común que caracteriza el profesorado, y éste se siente presionado para "motivar" a su alumnado en unas tareas que se denominan prácticas.

\footnotetext{
Es el caso de los ejemplos de hábitat disperso y el bocage en todo el norte de España, así como características tópicas como el individualismo, los prados y ganadería para todo un territorio. Una imagen que se repite en los manuales en décadas sucesivas, aun cuando el paisaje y las actividades se hayan modificado.

13 La descripción que hemos hecho se sustenta en la materia de geografía e historia de España del bachillerato de final del siglo XX, pero que perdura en las rutinas escolares del siglo XXI. Para más detalles de este modelo se puede consultar el libro de enseñanza de la geografía (Souto, 1999).
} 
Figura $\mathrm{N}^{0} 2$.

Síntesis del concepto de representaciones sociales

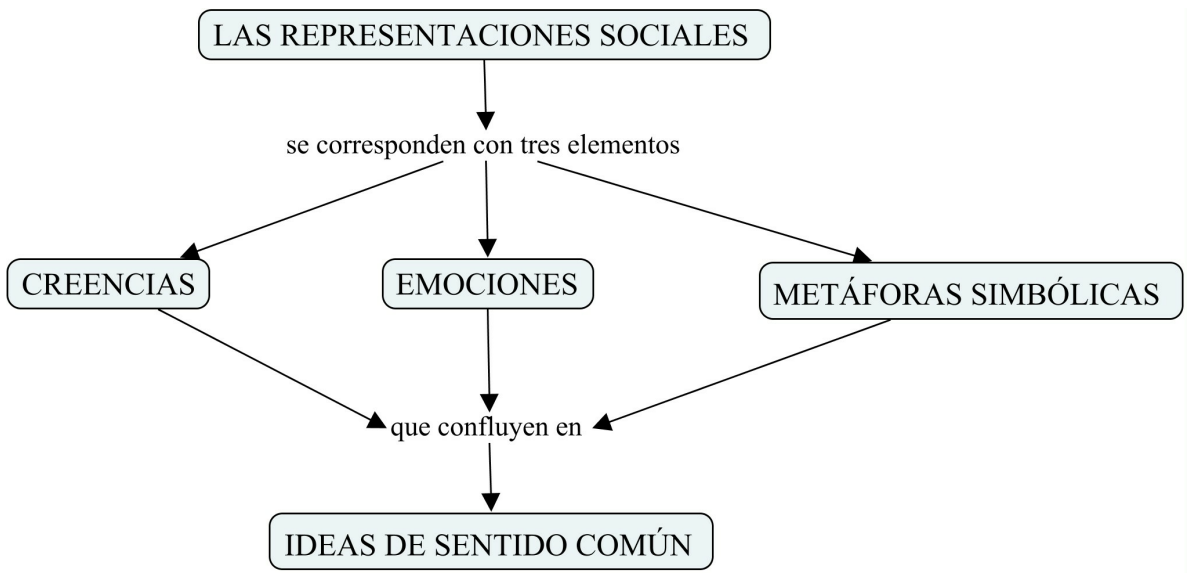

Fuente: Elaboración propia

Las representaciones sociales tienen una doble función. Por un lado, como modo de conocimiento o actividad de reproducción de las características de un objeto (reconstrucción mental); por otro lado, como una forma de pensamiento social que estructura la comunicación y las conductas de los miembros de un grupo. Finalmente, las representaciones sociales permiten que los sujetos construyan un pequeño modelo explicativo y evaluativo del entorno, a partir de un discurso, que le permite otorgar un sentido y le guía para funcionar en la vida social, contribuyendo en la resolución de problemas, conflictos y nuevas incertidumbres. Es una construcción dialéctica que se realiza en el contexto de las relaciones sociales, con las entradas de información procedentes de los medios de comunicación, de amigos, de familiares... tal como sucede en el caso de las representaciones territoriales analizadas por la Geografía de la Percepción y del Comportamiento.

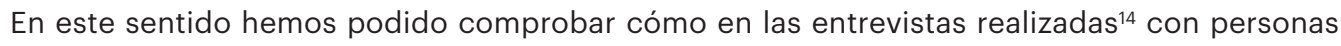
de diferentes edades ${ }^{15}$ (Cuadro $N^{0} 3$ ), se recordaba una clase de geografía escolar con una serie de continuidades, que no estaban lejos de la repetición memorística de un listado de nombres de lugares, en coincidencia con las opiniones expresadas por alumnos de Educación Secundaria de los años noventa del siglo pasado (Souto 1999: 65). Las diferencias que aparecían entre las personas de más edad y las de menos se concentraban más en aspectos de relación personal (profesor/alumno) y no en la materia que se estudiaba, lo que confirma trabajos anteriores en la enseñanza de la Historia de España (Souto, Martínez y Parra, 2014). Los recuerdos de agrado/ desagrado se transforman en fines para la enseñanza de la geografía en las expectativas de los futuros docentes.

\footnotetext{
Hasta enero de 2018 habíamos tabulado y analizado más de 300 entrevistas, que habían sido realizadas por el alumnado del Máster a personas en las tres cohortes de edad.

15 Las edades respondían a buscar una explicación de la geografía escolar dentro de las modificaciones legislativas más importantes en España: La LGE de 1970 y la LOGSE de 1990. Se pretendía validar la influencia de continuidades y cambios como consecuencia de las nuevas normativas legales.
} 
Cuadro No 3.

Percepción social de la geografía

\begin{tabular}{|c|c|c|c|}
\hline & $\begin{array}{c}\text { Menores } 30 \text { años } \\
(N=128)\end{array}$ & $\begin{array}{c}31 \text { a } 50 \text { años } \\
(N=98)\end{array}$ & $\begin{array}{c}\text { Mayores } 51 \text { años } \\
(\mathrm{N}=92)\end{array}$ \\
\hline $\begin{array}{l}\text { 1.-Recuerdo de } \\
\text { las clases de } \\
\text { geografía }\end{array}$ & $\begin{array}{l}\text { Profesores amenos y uso } \\
\text { de técnicas de represen- } \\
\text { tación } \\
\text { "El profesor fue exce- } \\
\text { lente. Contenidos y ma- } \\
\text { teriales adecuados, tan- } \\
\text { to el manual como las } \\
\text { transparencias, láminas } \\
\text { y mapas" }\end{array}$ & $\begin{array}{l}\text { Profesores pacientes y } \\
\text { uso del mapa. } \\
\text { "Haber estudiado las } \\
\text { capitales y los países } \\
\text { de los diferentes conti- } \\
\text { nentes". "Trabajar con } \\
\text { mapas "mudos", dibu- } \\
\text { jando ríos". }\end{array}$ & $\begin{array}{l}\text { Recuerdo negativo del } \\
\text { profesor } \\
\text { "te golpeaba con la vara } \\
\text { si errabas en la respues- } \\
\text { ta". "Aprender nombres } \\
\text { de lugares que estaban } \\
\text { en mapas". }\end{array}$ \\
\hline $\begin{array}{l}\text { 2.-Función de la } \\
\text { geografía }\end{array}$ & $\begin{array}{l}\text { Cultura general } \\
\text { "Sobre todo la demogra- } \\
\text { fía y geografía política" }\end{array}$ & $\begin{array}{l}\text { Comprender el mundo } \\
\text { y cultura general } \\
\text { "nos ayuda a entender } \\
\text { el porqué de muchas } \\
\text { cosas del mundo" }\end{array}$ & $\begin{array}{l}\text { Comprender el mundo } \\
\text { y cultura general } \\
\text { "saber dónde están los } \\
\text { lugares a los que nunca } \\
\text { se ha ido" }\end{array}$ \\
\hline $\begin{array}{l}\text { 3.-Elementos } \\
\text { necesarios } \\
\text { para ser buen } \\
\text { profesor de } \\
\text { geografía }\end{array}$ & $\begin{array}{l}\text { Técnicas de estudio } \\
\text { (memorización) } \\
\text { "Tener mucha memoria } \\
\text { y relacionar los conoci- } \\
\text { mientos con otras áreas } \\
\text { como historia, arte, po- } \\
\text { lítica..." }\end{array}$ & $\begin{array}{l}\text { Interés por la materia } \\
\text { "Estar motivado por la } \\
\text { disciplina y los conte- } \\
\text { nidos que nos puede } \\
\text { aportar" }\end{array}$ & $\begin{array}{l}\text { Capacidad de memori- } \\
\text { zación } \\
\text { "Tener memoria para } \\
\text { retener nombres" }\end{array}$ \\
\hline $\begin{array}{l}\text { 4.-Percepción } \\
\text { sobre el cambio } \\
\text { en la enseñanza } \\
\text { de la geografía }\end{array}$ & $\begin{array}{l}66.4 \text { \% (sí) } \\
\text { Nuevos temarios. Cam- } \\
\text { bios fronteras y TICs. }\end{array}$ & $\begin{array}{l}76 \% \text { (sí) } \\
\text { TICs y preparación do- } \\
\text { cente }\end{array}$ & $\begin{array}{l}84.2 \% \text { (sí) } \\
\text { Nuevos recursos y am- } \\
\text { pliación del temario } \\
\text { (escala local) }\end{array}$ \\
\hline
\end{tabular}

Fuente: Elaboración propia sobre encuestas realizadas a sujetos de los tres grupos de edad entre 2014 y 2017.

Estas entrevistas nos permitían comprobar que las respuestas escolares eran un reflejo de la opinión pública dominante en la sociedad, que activan las representaciones sociales y generan contradicciones aparentes derivadas de una dualidad entre la lógica cognitiva (racional y coherente) y la lógica social (irracional y e incoherente), que se manifiesta en frases docentes como ésta: "para lo que necesitan saber los niños y jóvenes, llega con conocer lo básico de la cultura universal y no la forma de elaborarse". Un pensamiento dominante que hemos impugnado desde la consideración profesional de la enseñanza de la geografía como una actividad que permite explicar y comprender los motivos por los cuales organizamos y usamos el medio de una determinada manera. 


\section{Las propuestas de solución: Ideas de las personas que quieren innovar}

Las representaciones sociales y la geografía de la percepción han puesto de relieve la incidencia de un sustrato inconsciente que nos determina los hábitos de pensamiento en relación con la enseñanza del espacio geográfico. Estas predisposiciones a actuar de una manera determinada, generalmente como reflejo de un guion docente del profesorado anterior, impide muchas veces mejorar la calidad de la enseñanza. Pero hay individuos y grupos que han buscado en la investigación educativa alternativas a esta manera de entender la didáctica escolar.

Dentro de colectivo de docentes de geografía e historia existen algunos sujetos que se han organizado para propiciar una alternativa innovadora a lo que se ha considerado como modelo hegemónico de la enseñanza de la Geografía. En España el número de personas que se han cuestionado la manera de ejercer la docencia de la Geografía es menor que en el caso de Historia, pues la procedencia de los docentes de Educación Secundaria es básicamente desde el ámbito institucional de Historia. Además el hecho de que la docencia en Educación Secundaria sea de ambas materias favorece la disgregación de los conocimientos geográficos en el discurso histórico.

Para poder conocer las posibilidades de cambio en la geografía escolar hemos analizado las opiniones y argumentos de las personas que formaban parte de los grupos de trabajo del proyecto Gea-Clío ${ }^{16}$. Para analizar sus opiniones hemos acudido a las siguientes fuentes de información, que nos permiten disponer de datos específicos (ver cuadro $\mathrm{n}^{\circ} 4$ ). Con dichos documentos, así como el contraste necesario que se ha establecido con sus producciones bibliográficas, hemos establecido algunas categorías conceptuales que nos permitirán concluir qué elementos forman parte de las innovaciones posibles en la enseñanza de la geografía.

Cuadro No 4.

Las evidencias de una alternativa educativa

\begin{tabular}{|l|l|}
\hline \multicolumn{1}{|c|}{ Fuentes documentales } & \multicolumn{1}{c|}{ Datos obtenidos } \\
\hline Grupo de discusión, 10 abril de 2013 & $\begin{array}{l}\text { Valoraciones sobre la innovación didáctica en el } \\
\text { área de geografía e historia }\end{array}$ \\
\hline Resumen entrevistas de 16 de mayo de 2016 & $\begin{array}{l}\text { Concepciones sobre los principios educativos } \\
\text { del grupo Gea-Clí, aportaciones conceptuales }\end{array}$ \\
\hline Entrevistas de octubre de 2016 & Aportaciones sobre el modelo docente \\
\hline $\begin{array}{l}\text { Artículo sobre Gea-Clío (Ramírez y Souto, } \\
2017 \text { ) }\end{array}$ & $\begin{array}{l}\text { Historia del proyecto educativo desde 1989 has- } \\
\text { ta } 2017\end{array}$ \\
\hline $\begin{array}{l}\text { Actas de las reuniones de grupo (desde } \\
2000 \text { hasta 2017) }\end{array}$ & $\begin{array}{l}\text { Preocupaciones, expectativas y realizaciones } \\
\text { del grupo Gea-Clío }\end{array}$ \\
\hline
\end{tabular}

Fuente: Elaboración propia con datos del archivo del proyecto Gea-Clío

El Grupo Gea-Clio nace en el curso escolar 1988-89 y permanece en activo en los momentos de redacción de este artículo (enero 2018). Su evolución histórica se puede analizar en el trabajo redactado por Santos Ramírez y Xosé M. Souto (2017). La búsqueda en google de Gea-Clío da 244.000 resultados (0,59 segundos). 
Hemos pretendido valorar si es posible impugnar las ideas dominantes en la enseñanza de la geografía, que se reduce a un aprendizaje repetitivo de nombres de diferentes escalas (ciudades, ríos, países, continentes) que "representan" la idea de cultura erudita. Además la influencia de las nuevas corrientes pedagógicas y las tecnologías de la información han incidido en generar una serie de tareas técnicas (hacer un climograma, consultar Google Maps...) que no están relacionadas con la resolución de problemas, sino que se realizan para "entretener". Todo ello genera una imagen enciclopédica del saber geográfico, que se organiza de manera yuxtapuesta en conceptos de difícil comprensión para el alumnado de 10 a 16 años de edad.

\section{Diagnóstico de la situación actual del profesorado de CC.SS. y Educación Primaria}

Los profesores que quieren innovar en enseñanza de la geografía entienden que uno de los obstáculos que influye en su trabajo es la característica específica de la docencia de una materia que tiene implicaciones morales, que se procuran racionalizar desde la ética y la epistemología. Una labor que implica un dominio conceptual de cuestiones filosóficas, pero también un compromiso social como manifiestan los profesores del colectivo Gea-Clío. Por eso se le concede una gran relevancia al rol de educador y a su capacidad de adaptarse a las condiciones sociales del entorno escolar; unas características profesionales que demandan una gran formación inicial. A continuación, mostramos algunas expresiones que avalan lo que estamos afirmando:

Hay otros profesores que, a mí me llamaba la atención, de CC.SS, pero también de Filosofía o Humanidades que huyen de la actualidad, porque esto son cuestiones que implican a la familia, (...), fomenta la argumentación, pero tomando partido y enseñando al alumnado a razonar argumentadamente y tomar partido de las situaciones. (PJO1, grupo de discusión de 10 de abril de 2013).

No encuentro la palabra o palabras exactas pero tendría que ser una persona que estuviera dispuesta a auto-exigirse ante los continuos problemas que plantee su alumnado. Esto supone ser autocrítico, formarse continuamente, crear expectativas y en definitiva estar dispuesto al cambio y a no dejar llevarse por las modas. (PCO2, octubre 2016).

Motivador: hem de treballar sempre per traure allò bo o positiu de cada alumne, reforçar els seus punts forts i animar-lo constantment a superar-se ${ }^{17}$. (PSO3, octubre 2016).

Para ello se reivindica un espacio curricular para la enseñanza de la geografía y de las ciencias sociales en el ámbito de la Educación Primaria ${ }^{18}$, pues se afirmaba que los docentes entendían que el contenido de geografía e historia no era relevante para el alumnado de estas edades. Por otra parte, los profesores/as que quieren innovar son conscientes del papel que tienen los libros de texto en la configuración de los programas escolares y por eso indican que un análisis que se hace

\footnotetext{
Los profesores del proyecto Gea-Clío utilizan los idiomas castellano y catalán para expresarse en sus reuniones. Hemos mantenido la expresión original y textual de sus palabras.

18 En España la Educación Primaria se organiza entre los seis y once años de edad (seis cursos escolares), donde el trabajo docente lo ejercen personas que se han diplomado o graduado en Magisterio sin especializarse en ciencias sociales. Por otra parte el contenido de Conocimiento del medio (que integra ciencias naturales y sociales) no se hacía de forma real, sino como yuxtaposición de materias. Desde 2014 ha desaparecido esta área, pero las ciencias sociales continúan con su propuesta enciclopédica y superficial.
} 
preciso no es sólo el contenido de los manuales, sino sobre todo el uso que se hace de ellos. En este sentido contamos con las valoraciones que los docentes han realizado del mismo proyecto Gea-Clío (Souto, 1996), en la cual subrayan la cantidad de trabajo necesaria para poder guiar el aprendizaje del alumnado con esta metodología; así destacaban la necesidad de cuestionar las rutinas referidas al tiempo escolar y a las valoraciones del mismo aprendizaje. Más recientemente hemos trabajado con más entrevistas y diarios de clase para poder profundizar en las ideas que poseen las personas que desean innovar, así como conocer los obstáculos que les afectan. Así podemos comprobar el uso superficial que se hace de la enseñanza de mapas, que como señalamos es un asunto repetido en la concepción dominante de esta materia:

... en cambio los alumnos vienen de $4^{\circ}, 5^{\circ}$ y $6^{\circ}$ (de Primaria)... vienen mapeados, han hecho mapas a punta pala. Sin embargo en $1^{\circ}$ (de Secundaria) no saben localizar las provincias, ríos... Porque, realmente, son añadidos, no son didácticos, cuando se trabajan en el aula no se trabajan de manera didáctica. (PSO4, grupo de discusión de 10 de abril de 2013).

Por eso, como señala un profesor (PA05), a los profesores/as les atemoriza cambiar sus rutinas para poder actuar con autonomía. Eso es lo que nos permite confirmar el diagnóstico inicial, pues son las concepciones y los miedos al cambio lo que dificultan dicha innovación:

Eso le produce pánico, entonces claro si tú tienes que salir del libro, esooo implica que vas a tener que pensar, vas a tener que repensar la clase y cómo vas a dar esa clase... y eso ya produce un vértigo... tremendo, $y$ entonces pasan.

(PA05, grupo de discusión de 10 de abril de 2013).

Todo ello supone la complejidad de la formación del profesorado de geografía e historia ${ }^{19}$, pues como se señala por el profesorado de este proyecto curricular se necesitan conocimientos epistemológicos, psicopedagógicos, curriculares y sociológicos, que determina las características ideales de la profesión docente. Una definición que es compleja desde la perspectiva teórica y más desde la praxis escolar, determinada por las normativas institucionales. Por eso las dudas de la labor docente sirven de estímulo más que corresponden a una descripción de la personalidad, tal y como recogen estas respuestas seleccionadas de las actas de una asamblea ordinaria del proyecto Gea Clío:

-para su trabajo en el aula y con alumnado sería necesario conocer con suficiencia las tres disciplinas de referencia -Historia, Geografía e Historia del Arte- especialmente en su dimensión epistemológica.

-qué suponen como forma de pensar geográficamente o históricamente; también se necesitaría conocer aquellos conocimientos básicos psicopedagógicos de naturaleza procedimental y comunes a las ciencias de la educación.

19 En España el profesorado de Educación Secundaria (12 a 18 años de edad del alumnado) comparte las materias de Geografía, Historia e Historia del Arte, que tienen una formación inicial diferente. En la Educación Primaria (6 a 11 años de edad) comparten todas las materias de ciencias y se forman en las facultades de educación. 
-para su trabajo fuera del aula en centros se requeriría conocer el funcionamiento básico curricular y técnico de la educación secundaria.

-el entorno (medio), las relaciones sociales establecidas entre todos sus componentes y las nociones teóricas y conceptos necesarios para poder entenderlas.

En el mundo de hoy, lo que les sobra es información. Lo que no tienen son criterios ni herramientas para lidiar con las montañas de información con las que son bombardeados todos los días.

(Actas de Gea-Clío. Noviembre 2016)

\section{Conclusiones}

En este artículo hemos pretendido analizar los estereotipos que se aceptan en la enseñanza de la geografía en el sistema escolar. Para ello hemos acudido a la teoría de las representaciones sociales, pues tal como sucede con la Geografía de la Percepción y del Comportamiento, nos permite explicar y comprender las relaciones que se establecen entre las decisiones personales y la visión del mundo. Ponen en relación los recuerdos personales con las explicaciones históricas en una simbiosis que les hace resistentes a las nuevas informaciones que se suministran, o bien asimilan los datos escolares en una previa explicación del mundo que implica unas determinadas decisiones individuales. En los estudios empíricos realizados hemos constatado la incidencia de los recuerdos de las clases que se habían recibido cuando los futuros docentes eran niños y adolescentes. Por eso buscan en actividades prácticas y lúdicas una solución a su valoración negativa de la enseñanza de la geografía, que se aleja así de una reflexión consciente sobre la praxis escolar.

En este sentido resulta muy ilustrativa la concepción de la geografía que tienen los profesores actuales y futuros, tal como hemos indicado. Su referencia a la asociación de los mapas con la enseñanza del espacio geográfico no va acompañada de un estudio crítico de las representaciones cartográficas. Así se acepta como "natural" la representación del mundo de Mercator, una cartografía que supone que el hemisferio Norte ocupa las dos terceras partes del mapamundi y que Groenlandia que es casi tres veces más pequeña que Brasil aparezca más grande. Y, sobre todo, se ofrece una visión eurocéntrica del mundo, donde Europa hasta los Urales aparece con una representación superficial casi tan grande como América del Sur, pese a que sea la mitad. Y ello lo hemos observado en los manuales escolares de Brasil, Colombia... o sea, los países de América del Sur adoptan una cartografía "imperialista" cuando en muchas manifestaciones públicas se rebelan contra ella.

Por eso hemos observado que las ideas del sentido común encierran una representación del mundo que es contradictoria con la ideología que se dice defender. Y ello es un ejemplo característico de su potencial educativo. Nos permite cuestionar la realidad observada, para lo cual hemos venido defendiendo desde hace tiempo la necesidad de un estudio epistemológico de la geografía, que cuestione la cosmovisión de los manuales escolares y las informaciones de los medios de comunicación. Pero siendo necesario este conocimiento no es suficiente. Es preciso analizar el contenido didáctico del objeto del saber, en este caso los hechos que se transmiten 
desde la ciencia geográfica. Se necesita conocer que el espacio geográfico se construye en la interacción entre el espacio vivido de las emociones y el espacio absoluto de las concepciones sociales del poder político. Entre ambas aparece el espacio complejo de la Geografía de la Percepción y del Comportamiento.

En este sentido hemos de precisar las relaciones entre el espacio cotidiano y el espacio escolar. El primero debe ser diferenciado en su contexto emocional y en su ámbito de las relaciones sociales (las percepciones espaciales), mientras que el escolar está teñido de las concepciones geográficas, que aspiran a mantener un orden social desde la sutileza de procesos de poder. Una síntesis que influye en la representación social del mundo de los futuros docentes (Morales et al., 2014).

Y para ello, como anotan los miembros de Gea-Clío, es preciso disponer de principios educativos filosóficos y un buen conocimiento del currículo y del marco social donde se desarrolla la labor docente. Una magna empresa para la que es preciso organizarse colectivamente. Los ejemplos de las producciones bibliográficas que han sido sometidas a evaluación pública ${ }^{20}$, nos permiten verificar que las alternativas educativas son posibles, pero que necesitan constancia, rigor y cuestionamiento de la realidad aparente.

\section{Referencias bibliográficas}

ABRIC, J.C. Méthodologie de recueil des représentations sociales. En: ABRIC, J.C. Pratiques Sociales et représentations. París: PUF, 1994, p. 59-82.

BACHELARD, G. La formación del espíritu científico. México: Editorial Siglo XXI, 1987.

BEL MARTÍNEZ, J.C.; PORTOLÉS PORCAR, S.; BAYÓ JAIME, M. y RAMIRO ROCA, E. El profesorado de geografía en Perú y su percepción de los estados americanos y europeos: una aproximación a las representaciones sociales en los docentes. Revista de Didácticas Específicas, 2017, N016, p. 96-112.

BOIRA, J.V. y REQUES, P. Introducción al Estudio de la Percepción Espacial. Principado de Andorra: Consejería de Educación de los Centros Docentes Españoles, 1991.

BOURDIEU, P. y PASSERON, J. C. La reproducción. Elementos para una teoría del sistema de enseñanza. Barcelona: Editorial Laia, 1979.

CAPEL, H. Percepción del medio y comportamiento geográfico. Revista de Geografía, 1973, No 7, p. 58-150.

En la página web socialsuv.org en la pestaña Gea-Clío se puede visualizar en abierto y libre la producción de los materiales didácticos de este grupo. 
CAPEL, H.; ARAYA, M.; BRUNET, M.; COLLEL, A.; MELCÓN, J. y NADAL, F. Ciencia para la burguesía. Renovación pedagógica y enseñanza de la geografía durante la Revolución liberal (1814-1857). Barcelona: Universidad de Barcelona, 1983.

CAPEL, H.; CAMPS, E.; DEL CASTILLO, M.A.; MAYANS, B.; MELENDO, M.I.; PERICÁS, C.; RIBA, P.; RISPA, J.M. y SANS, M. Geografía para todos. La geografía en la enseñanza española durante la segunda mitad del siglo XIX. Barcelona: Los Libros de la Frontera, 1985.

CARABAÑA, J. La inutilidad de PISA para las escuelas. Madrid: Los libros de la Catarata, 2015.

CARRETERO, M.; JACOTT, L.; LIMÓN, M. y LÓPEZ-MANJÓN, A. Construir y enseñar. Las Ciencias Sociales y la Historia. Madrid: Visor, 1995.

COLL, C.; MARTÍN, E.; MAURI, T.; ONRUBIA, J.; SOLÉ, I. y ZABALA, A. El constructivismo en el aula. Barcelona: Graó, 1993.

CUESTA, R. Sociogénesis de una disciplina escolar: la Historia. Barcelona: Pomares-Corredor, 1997.

DAMASIO, A. Y el cerebro creó al hombre. Barcelona: Destino, 2010.

DÍAZ, J.L. La conciencia viviente. México: Fondo de Cultura Económica, 2007.

DOMINGOS, M. Habitus e representações sociais: questões para o estudo de identidades coletivas. En: MOREIRA, A. y OLIVEIRA, D.C. Estudos interdisciplinares de representação social. Goiânia: $A B, 2000, N^{\circ} 200$, p. 117-130.

DOMINGOS, M. Representações Sociais como obstáculos Simbólicos à Incorporação do Habitus Científico. Ariús, 2010, № 2, p. 31-48.

DRIVER, R. Un enfoque constructivista para el desarrollo del currículo en ciencias. Enseñanza de las ciencias: revista de investigación y experiencias didácticas, 1988, No 6 (2), p. 109-120.

FERNÁNDEZ, M.V.; GUVERICH, R.; SOUTO, P.; BACHMANN, L.; AJÓN, A. y QUINTERO, S. La imagen pública de la Geografía, una indagación desde las visiones de profesores y padres de alumnos secundarios. Biblio 3W, Revista Bibliográfica de Geografía y Ciencias Sociales, 2010, vol. XV, no 859.

FRAILE-JURADO, P. y LÓPEZ-MARTÍN, M. Mitos geográficos contemporáneos. El conocimiento geográfico reglado frente al fake science en el contexto 2.0. Ar@cne. Revista Electrónica de Recursos de Internet sobre Geografía y Ciencias Sociales. Barcelona: Universidad de Barcelona, 2017, No 222.

GIL-PÉREZ, D. Diez años de investigación en didáctica de las ciencias: realizaciones y perspectivas. Enseñanza de las Ciencias, 1994, Vol. 12 (2), p. 154-164. 
GOODSON, I.F. Historia del Currículum. La construcción social de las disciplinas escolares. Barcelona: Pomares-Corredor, 1995.

GUSTAVSSON, A. \& SELANDER, S. Transformations and Changes in Social Knowledge: Towards the Dynamics of Meaning Making. In: CHAIB, M.; DANERMARK, B. Y SELANDER, S. Education, Professionalization and Social Representation. New York: Routledge, 2011, p. 17-32.

JODELET, D. Representações sociais: um domínio em expansão. En: JODELET, D. As Representações Sociais. Rio de Janeiro: EdUERJ, 2001.

LUIS GÓMEZ, A. La Geografía en el bachillerato español (1836-1970). Barcelona: Publicaciones de la Universidad de Barcelona, 1985.

MELCÓN BELTRÁN, J. Renovación de la enseñanza de la geografía en los orígenes de la España contemporánea. Madrid: Universidad Autónoma de Madrid, 1995.

MORALES, A.J; CAURIN, C.; y SOUTO, X.M. Percepción del mundo: mapas mentales y problemas socioambientales. Didáctica Geográfica, 2013, №14, p. 91-108.

MOSCOVICI, S. El psicoanálisis, su imagen y su público. Buenos Aires: Huemul S.A., 1979.

MOSCOVICl, S. A invenção da sociedade. Sociologia e Psicologia. Petrópolis: Editora Vozes, 2011.

MÜLLER, A. ¿Qué queda de la Reforma? Terra, 1993, № 5, p. 22-25.

PALACIOS, N. La experiencia social de la educación. Un estudio de tres instituciones educativas de secundaria en Colombia, Bogotá: Ediciones Uniandes, 2018.

PALACIOS, N. y HERRERA, J. D. Subjetividad, socialización política y derechos en la escuela. Magis, Revista Internacional de Investigación en Educación, 2013, No 5 (11), p. 413-437.

PÉREZ, A. y SOUTO, X.M. The curriculum framework and the teaching of Geography in Spain. The European Geographer, 1989, № 2, p. 36-40.

RAMÍREZ, S. y SOUTO, X.M. Gea-Clío. La praxis escolar para mejorar la autonomía ciudadana. En: GARCÍA-MONTEAGUDO, D.; RAMÍREZ, S. y SOUTO. X.M. Las buenas praxis escolares: investigar desde la práctica de aula. Valencia: Nau Llibres, 2017, p. 153-177.

RAMIRO, E. Institucionalització i difusió de la Geografia Escolar: la Percepció del professorat de la comarca de la Ribera. Tesis doctoral. Valencia: Universitat de València, 1998.

RANGEL, H. T. Inteligencia, Competencias y Constructivismo. Más Allá de la Teoría de Gardner. Revista Mexicana de Investigación en Psicología, 2010, Vol. 2, No (2), p. 141-146. 
SARAIVA, J. Habitus docente e representaçao social do ensinar geografia na Educaçao Básica de Teresina-Piauí. Natal: Programa Posgraduaçao em Educaçao, Universidade Federal Río Grande do Norte, 2007.

SATURNINO, J. ¿Baja el nivel educativo? Madrid: Diario El País, 2005.

SOUTO, X.M.; MARTÍNEZ, N. y PARRA, D. La representación poliédrica de la memoria escolar. Un proyecto de investigación sobre las clases de Historia de España. En: MARTíNEZ, N. La Historia de España en los recuerdos escolares. Análisis, interpretación y poder de cambio de los testimonios de profesores y alumnos. Valencia: Nau Llibres, 2014, p. 29-52.

SOUTO, X.M. Investigación educativa y experimentación del proyecto curricular de geografía GEA. En: Grupo de Didáctica de las Ciencias Sociales del Proyecto IRES. La experimentación curricular en ciencias sociales: planteamientos y perspectivas. Sevilla: Alfar, 1996, p. 79-96.

SOUTO, X.M. Didáctica de la Geografía, Problemas sociales y Conocimiento del medio. Barcelona: Ediciones del Serbal, 1999.

SOUTO, X.M. Investigación e innovación educativa: el caso de la Geografía escolar. Scripta Nova. Revista Electrónica de Geografía y Ciencias Sociales. Barcelona: Universidad de Barcelona, 2013, Vol. XVII, No 459. Disponible en: <http://www.ub.es/geocrit/sn/sn-459.htm>. 